\title{
Interacting agegraphic dark energy models in non-flat universe
}

\author{
Ahmad Sheykhi * \\ Department of Physics, Shahid Bahonar University, P.O. Box 76175, Kerman, Iran \\ Research Institute for Astronomy and Astrophysics of Maragha (RIAAM), Maragha, Iran
}

\begin{abstract}
A so-called "agegraphic dark energy", was recently proposed to explain the dark energy-dominated universe. In this Letter, we generalize the agegraphic dark energy models to the universe with spatial curvature in the presence of interaction between dark matter and dark energy. We show that these models can accommodate $w_{D}=-1$ crossing for the equation of state of dark energy. In the limiting case of a flat universe, i.e. $k=0$, all previous results of agegraphic dark energy in flat universe are restored.
\end{abstract}

\section{INTRODUCTION}

The dark energy problem constitute a major puzzle of modern cosmology. A great variety of cosmological observations suggest that our universe is currently undergoing a phase of accelerated expansion likely driven by some unknown energy component whose main feature is to possess a negative pressure [1]. Although the nature of such dark energy is still speculative, an overwhelming flood of papers has appeared which attempt to describe it by devising a great variety of models. Among them are cosmological constant, exotic fields such as phantom or quintessence, modified gravity, etc, see [2] for a recent review. Recently, a new dark energy candidate, based not in any specific field but on the holographic principle, was proposed [3, 3 ]. According to the holographic principle, the number of degrees of freedom of physical systems scale with their bounding area rather than with their volume 5]. On these basis, Cohen et al. 6] suggested that in quantum field theory a short distance cutoff is related to a long distance cutoff due to the limit set by formation of a black hole, which results in an upper bound on the zero-point energy density. The extension of the holographic principle to a general cosmological setting was addressed by Fischler and Susskind 77]. Following this line, Li [8] argued that zero-point energy density could be viewed as the holographic dark energy density satisfying $\rho_{D} \leq 3 c^{2} m_{p}^{2} / L^{2}$, the equality sign holding only when the holographic bound is saturated. Here $c^{2}$ is a constant, the coefficient 3 is for convenience, $L$ is an IR cutoff and $m_{p}^{2}=(8 \pi G)^{-1}$. The holographic models of dark energy have been proposed and studied widely in the literature [9, 10, 11, 12]. It is fair to claim that simplicity and reasonability of holographic model of dark energy provides more reliable frame to investigate the problem of dark energy rather than other models proposed in the literature [13]. For example the coincidence problem can be easily solved in some models of holographic dark energy [14.

More recently, a new dark energy model, called agegraphic dark energy (ADE) was proposed by Cai [15].

\footnotetext{
*sheykhi@mail.uk.ac.ir
}

This model is based on the uncertainty relation of quantum mechanics together with the gravitational effect in general relativity. Following the line of quantum fluctuations of spacetime, Karolyhazy et al. [16] argued that the distance $t$ in Minkowski spacetime cannot be known to a better accuracy than $\delta t=\beta t_{p}^{2 / 3} t^{1 / 3}$ where $\beta$ is a dimensionless constant of order unity. Based on Karolyhazy relation, Maziashvili discussed that the energy density of metric fluctuations of the Minkowski spacetime is given by 17 ]

$$
\rho_{D} \sim \frac{1}{t_{p}^{2} t^{2}} \sim \frac{m_{p}^{2}}{t^{2}}
$$

where $t_{p}$ is the reduced Planck time. Throughout this Letter we use the units $c=\hbar=k_{b}=1$. Therefore one has $l_{p}=t_{p}=1 / m_{p}$ with $l_{p}$ and $m_{p}$ are the reduced Planck length and mass, respectively. The agegraphic dark energy model assumes that the observed dark energy comes from the spacetime and matter field fluctuations in the universe [18, 19]. The agegraphic models of dark energy have been examined [20, 21, 22] and constrained by various astronomical observations [23].

On the other hand, lacking a fundamental theory, most discussions on dark energy rely on the fact that its evolution is independent of other matter fields. Given the unknown nature of both dark matter and dark energy there is nothing in principle against their mutual interaction and it seems very special that these two major components in the universe are entirely independent. Indeed, this possibility is receiving growing attention in the literature [24, 25] (see also [26, 27] and references therein) and appears to be compatible with SNIa and CMB data [28]. Furthermore, the interacting holographic dark energy models have also been extended to the universe with spacial curvature [13, 29].

Besides, it is generally believed that inflation practically washes out the effect of curvature in the early stages of cosmic evolution. However, it does not necessarily imply that the curvature has to be wholly neglected at present. Indeed, aside from the sake of generality, there are sound reasons to include it: (i) Inflation drives the $k / a^{2}$ ratio close to zero but it cannot set it to zero if $k \neq 0$ initially. (ii) The closeness to perfect flatness depends on the number of e-folds and we can only speculate about the latter. (iii) After inflation the absolute 
value of the $k / a^{2}$ term in the field equations may increase with respect to the matter density term, thereby the former should not be ignored when studying the late universe. (iv) Observationally there is room for a small but non-negligible spatial curvature [30]. For instance, the tendency of preferring a closed universe appeared in a suite of CMB experiments [31]. The improved precision from WMAP provides further confidence, showing that a closed universe with positively curved space is marginally preferred [32]. In addition to CMB, recently the spatial geometry of the universe was probed by supernova measurements of the cubic correction to the luminosity distance [33], where a closed universe is also marginally favored.

In the light of all mentioned above, it becomes obvious that the investigation on the interacting agegraphic dark energy models in the universe with spacial curvature is well motivated. In this Letter, we would like to generalize, following [13], the agegraphic dark energy models to the universe with spacial curvature in the presence of interaction between the dark matter and dark energy. We will also show that the equation of state of dark energy can accommodate $w=-1$ crossing. The plan of the work is as follows: In section 1 we study the original agegraphic model of dark energy in a non-flat universe where the time scale is chosen to be the age of the universe. In section IIII we consider the new model of agegraphic dark energy while the time scale is chosen to be the conformal time instead of the age of the universe. Finally, in section IV we summarize our results.

\section{THE ORIGINAL ADE IN NONFLAT UNIVERSE}

The original agegraphic dark energy density has the form (11) where $t$ is chosen to be the age of the universe

$$
T=\int d t=\int_{0}^{a} \frac{d a}{H a}
$$

where $a$ is the scale factor and $H=\dot{a} / a$ is the Hubble parameter. Thus, the energy density of the agegraphic dark energy is given by [15]

$$
\rho_{D}=\frac{3 n^{2} m_{p}^{2}}{T^{2}}
$$

where the numerical factor $3 n^{2}$ is introduced to parameterize some uncertainties, such as the species of quantum fields in the universe, the effect of curved space-time (since the energy density is derived for Minkowski spacetime), and so on. The dark energy density (3) has the same form as the holographic dark energy, but the length measure is chosen to be the age of the universe instead of the horizon radius of the universe. Thus the causality problem in the holographic dark energy is avoided [15].

The total energy density is $\rho=\rho_{m}+\rho_{D}$, where $\rho_{m}$ and $\rho_{D}$ are the energy density of dark matter and dark energy, respectively. The total energy density satisfies a conservation law

$$
\dot{\rho}+3 H(\rho+p)=0 .
$$

However, since we consider the interaction between dark matter and dark energy, $\rho_{m}$ and $\rho_{D}$ do not conserve separately; they must rather enter the energy balances

$$
\begin{aligned}
& \dot{\rho}_{m}+3 H \rho_{m}=Q, \\
& \dot{\rho}_{D}+3 H \rho_{D}\left(1+w_{D}\right)=-Q .
\end{aligned}
$$

Here $w_{D}$ is the equation of state parameter of agegraphic dark energy and $Q$ denotes the interaction term and can be taken as $Q=3 b^{2} H \rho$ with $b^{2}$ being a coupling constant. This expression for the interaction term was first introduced in the study of the suitable coupling between a quintessence scalar field and a pressureless cold dark matter field [24]. In the context of holographic dark energy model, this form of interaction was derived from the choice of Hubble scale as the IR cutoff [14]. Although at this point the interaction may look purely phenomenological but different Lagrangians have been proposed in support of it (see [34] and references therein). Besides, in the absence of a symmetry that forbids the interaction there is nothing, in principle, against it. Further, the interacting dark materdark energy (the latter in the form of a quintessence scalar field and the former as fermions whose mass depends on the scalar field) has been investigated at one quantum loop with the result that the coupling leaves the dark energy potential stable if the former is of exponential type but it renders it unstable otherwise [35]. Thus, microphysics seems to allow enough room for the coupling; however, this point is not fully settled and should be further investigated. The difficulty lies, among other things, in that the very nature of both dark energy and dark matter remains unknown whence the detailed form of the coupling cannot be elucidated at this stage.

For the Friedmann-Robertson-Walker (FRW) universe filled with dark energy and dust (dark matter), the corresponding Friedmann equation takes the form

$$
H^{2}+\frac{k}{a^{2}}=\frac{1}{3 m_{p}^{2}}\left(\rho_{m}+\rho_{D}\right),
$$

where $k$ is the curvature parameter with $k=-1,0,1$ corresponding to open, flat, and closed universes, respectively. A closed universe with a small positive curvature $\left(\Omega_{k} \simeq 0.02\right)$ is compatible with observations [30]. If we introduce, as usual, the fractional energy densities such as

$$
\Omega_{m}=\frac{\rho_{m}}{3 m_{p}^{2} H^{2}}, \quad \Omega_{D}=\frac{\rho_{D}}{3 m_{p}^{2} H^{2}}, \quad \Omega_{k}=\frac{k}{H^{2} a^{2}},
$$

then the Friedmann equation can be written

$$
\Omega_{m}+\Omega_{D}=1+\Omega_{k} .
$$

Using Eq. (3), we have

$$
\Omega_{D}=\frac{n^{2}}{H^{2} T^{2}} .
$$


Differentiating Eq. (10) and using relation $\dot{\Omega}_{D}=\Omega_{D}^{\prime} H$, we reach

$$
\Omega_{D}^{\prime}=\Omega_{D}\left(-2 \frac{\dot{H}}{H^{2}}-\frac{2}{n} \sqrt{\Omega_{D}}\right),
$$

where the dot is the derivative with respect to the cosmic time and the prime denotes the derivative with respect to $x=\ln a$. Taking the derivative of both side of the Friedman equation (7) with respect to the cosmic time, and using Eqs. (3), (5) , (9) and (10), it is easy to find that

$$
\frac{\dot{H}}{H^{2}}=-\frac{3}{2}\left(1-\Omega_{D}\right)-\frac{\Omega_{D}^{3 / 2}}{n}-\frac{\Omega_{k}}{2}+\frac{3}{2} b^{2}\left(1+\Omega_{k}\right) .
$$

Substituting this relation into Eq. (11), we obtain the equation of motion of agegraphic dark energy

$$
\begin{aligned}
\Omega_{D}^{\prime}= & \Omega_{D}\left[\left(1-\Omega_{D}\right)\left(3-\frac{2}{n} \sqrt{\Omega_{D}}\right)\right. \\
& \left.-3 b^{2}\left(1+\Omega_{k}\right)+\Omega_{k}\right] .
\end{aligned}
$$

Inserting $\Omega_{k}=0=b$, this equation reduces to Eq. (12) of Ref. [15]. Using Eqs. (3) and (6), as well as Eq. (10), we can obtain the equation of state parameter for the interacting agegraphic dark energy

$$
w_{D}=-1+\frac{2}{3 n} \sqrt{\Omega_{D}}-b^{2} \Omega_{D}^{-1}\left(1+\Omega_{k}\right) .
$$

The total equation of state parameter is given by

$$
w_{\text {tot }}=\frac{p}{\rho}=\frac{\Omega_{D}}{1+\Omega_{k}} w_{D}
$$

For completeness, we give the deceleration parameter

$$
q=-\frac{\ddot{a}}{a H^{2}}=-1-\frac{\dot{H}}{H^{2}},
$$

which combined with the Hubble parameter and the dimensionless density parameters form a set of useful parameters for the description of the astrophysical observations. Substituting Eq. (12) in Eq. (16) we get

$$
q=\frac{1}{2}-\frac{3}{2} \Omega_{D}+\frac{\Omega_{D}^{3 / 2}}{n}-\frac{3}{2} b^{2}+\frac{1}{2} \Omega_{k}\left(1-3 b^{2}\right) .
$$

It is worth noting that in the absence of interaction between dark energy and dark matter, $b^{2}=0$, from Eq. (14) we see that $w_{D}$ is always larger than -1 and cannot cross the phantom divide $w_{D}=-1$. In addition the condition $n>1$ is necessary to derive the present accelerated expansion [15]. However, the situation is changed as soon as the interaction term is taken into account. In this case $\left(b^{2} \neq 0\right)$, from Eq. (14) one can easily see that $w_{D}$ can cross the phantom divide provided $3 n b^{2}\left(1+\Omega_{k}\right)>2 \Omega_{D}^{3 / 2}$. If we take $\Omega_{D}=0.72$ and $\Omega_{k}=0.02$ for the present time, the phantom-like equation of state can be achieved only if $n b^{2}>0.4$. The best fit result for agegraphic dark energy which is consistent with most observations like WMAP and SDSS data, shows that $n=3.4$ [23]. Thus, the condition $w_{D}<-1$ leads to $b^{2}>0.12$ for the coupling between dark energy and dark matter. For instance, if we take $b^{2}=0.15$ we get $w_{D}=-1.05$. This indicates that one can generate phantom-like equation of state from an interacting agegraphic dark energy model in the universe with any spacial curvature. Putting $\Omega_{k}=0$ in Eqs. (14) and (17), these equations reduce to their respective equations of original interacting agegraphic dark energy model in flat universe [18]. The original interacting agegraphic dark energy has laso some interesting features. From Eq. (14), it is easy to see that $w_{D}<-1$ is necessary in the early time where $\Omega_{D} \rightarrow 0$, while in the late time where $\Omega_{D} \rightarrow 1\left(\Omega_{k} \simeq 0\right)$, we have $w_{D}>-1$ for $n b^{2}<0.67$ and $w_{D}<-1$ for $n b^{2}>0.67$.

It is important to note that in the absence of interaction, the original agegraphic dark energy model has a drawback to describe the matter-dominated universe in the far past where $a \ll 1$ and $\Omega_{D} \ll 1$. On one side, from Eq. (14) with $b^{2}=0$ we have $w_{D} \rightarrow-1$ as $\Omega_{D} \rightarrow 0$. This means that in the matter-dominated epoch the agegraphic dark energy behaves like a cosmological constant. On the other side, Eq. (13) with $b^{2}=0, \Omega_{D} \ll 1$ and $\Omega_{k} \ll 1$ approximately becomes

$$
\frac{d \Omega_{D}}{d a} \simeq \frac{\Omega_{D}}{a}\left(3-\frac{2}{n} \sqrt{\Omega_{D}}\right)
$$

which has a solution of the form $\Omega_{D}=9 n^{2} / 4$. Substituting this relation into Eq. (14) with $b^{2}=0$, we get $w_{D}=0$. Therefore, the dark energy behaves as pressureless matter. Obviously, pressureless matter cannot generate accelerated expansion, which seems to rule out the choice $t=T$. Thus there is a confusion in the original agegraphic dark energy model. This issue is similar to the holographic dark energy model when choosing the Hubble parameter as the IR cutoff [4]. It was argued by Pavon and Zimdahl that the problem can be solved as soon as an interaction between the dark energy and dark matter is taken into account [14]. Similarly, in the agegraphic model of dark energy the inconsistency in the original version can be removed with the interaction between dark energy and dark matter. To see this, consider the matter-dominated epoch where $a \ll 1$ and $\Omega_{D} \ll 1$ for interacting agegraphic dark energy. In this case Eq. (13) with $\Omega_{k} \ll 1$ approximately becomes

$$
\frac{d \Omega_{D}}{d a} \simeq \frac{\Omega_{D}}{a}\left(3-\frac{2}{n} \sqrt{\Omega_{D}}-3 b^{2}\right),
$$

which has a solution of the form $\Omega_{D}=9 n^{2}\left(1-b^{2}\right)^{2} / 4$. Substituting this relation into Eq. (14), we obtain

$$
w_{D}=-b^{2}\left(1+\frac{4}{9 n^{2}\left(1-b^{2}\right)^{2}}\right) .
$$

Therefore for $b \neq 0$ we have $w_{D}<0$, and the agegraphic model of dark energy can generate accelerated expansion. 
The presence of the spatial curvature does not seriously modify the above discussion. Thus, the confusion in the original agegraphic dark energy model without interaction is removed. Nevertheless, Wei and Cai [19] proposed a new model of agegraphic dark energy to resolve the contradiction in the far past of original non-interacting agegraphic dark energy model.

\section{THE NEW MODEL OF ADE IN NONFLAT UNIVERSE}

As we argued the original agegraphic dark energy model has some difficulties [15]. Therefore one may seek for another agegraphic dark energy model. Wei and Cai proposed a new model of agegraphic dark energy [19], while the time scale is chosen to be the conformal time $\eta$ instead of the age of the universe, which is defined by $d t=a d \eta$, where $t$ is the cosmic time. It is worth noting that the Karolyhazy relation $\delta t=\beta t_{p}^{2 / 3} t^{1 / 3}$ was derived for Minkowski spacetime $d s^{2}=d t^{2}-d \mathrm{x}^{2}$ [16, 17]. In the case of the FRW universe, we have $d s^{2}=d t^{2}-a^{2} d \mathrm{x}^{2}=a^{2}\left(d \eta^{2}-d \mathrm{x}^{2}\right)$. Therefore, it is more reasonable to choose the time scale in Eq. (3) to be the conformal time $\eta$ [19]. Taking this into account, the energy density of the new agegraphic dark energy can be written

$$
\rho_{D}=\frac{3 n^{2} m_{p}^{2}}{\eta^{2}}
$$

where the conformal time is given by

$$
\eta=\int \frac{d t}{a}=\int_{0}^{a} \frac{d a}{H a^{2}} .
$$

If we write $\eta$ to be a definite integral, there will be an integral constant in addition. Thus, we have $\dot{\eta}=1 / a$. Let us again consider a FRW universe with spatial curvature containing the new agegraphic dark energy and pressureless matter. The Friedmann equation can be written

$$
H^{2}+\frac{k}{a^{2}}=\frac{1}{3 m_{p}^{2}}\left(\rho_{m}+\rho_{D}\right),
$$

where can be rewritten as

$$
\Omega_{m}+\Omega_{D}=1+\Omega_{k} .
$$

The fractional energy density of the agegraphic dark energy is now given by

$$
\Omega_{D}=\frac{n^{2}}{H^{2} \eta^{2}}
$$

We can also find the equation of motion for $\Omega_{D}$ by taking the derivative of Eq. (25). The result is

$$
\Omega_{D}^{\prime}=\Omega_{D}\left(-2 \frac{\dot{H}}{H^{2}}-\frac{2}{n a} \sqrt{\Omega_{D}}\right)
$$

Taking the derivative of both side of the Friedman equation (23) with respect to the cosmic time $t$, and using Eqs. (5), (21), (24) and (25), we obtain

$$
\frac{\dot{H}}{H^{2}}=-\frac{3}{2}\left(1-\Omega_{D}\right)-\frac{\Omega_{D}^{3 / 2}}{n a}-\frac{\Omega_{k}}{2}+\frac{3}{2} b^{2}\left(1+\Omega_{k}\right) .
$$

Substituting this relation into Eq. (26), we obtain the evolution behavior of the new agegraphic dark energy

$$
\begin{aligned}
\Omega_{D}^{\prime}= & \Omega_{D}\left[\left(1-\Omega_{D}\right)\left(3-\frac{2}{n a} \sqrt{\Omega_{D}}\right)\right. \\
& \left.-3 b^{2}\left(1+\Omega_{k}\right)+\Omega_{k}\right] .
\end{aligned}
$$

The equation of state parameter of the interacting new agegraphic dark energy can be obtained as

$$
w_{D}=-1+\frac{2}{3 n a} \sqrt{\Omega_{D}}-b^{2} \Omega_{D}^{-1}\left(1+\Omega_{k}\right),
$$

Again we see that $w_{D}$ can cross the phantom divide provided $3 n a b^{2}\left(1+\Omega_{k}\right)>2 \Omega_{D}^{3 / 2}$. Taking $\Omega_{D}=0.72$, $\Omega_{k}=0.02$, and $a=1$ for the present time, the phantomlike equation of state can be achieved only if $n b^{2}>0.4$. The joint analysis of the astronomical data for the new agegraphic dark energy gives the best-fit value (with $1 \sigma$ uncertainty) $n=2.7$ [23]. Thus, the condition $w_{D}<-1$ leads to $b^{2}>0.15$ for the coupling between dark energy and dark matter. For instance, if we take $b^{2}=0.2$ we get $w_{D}=-1.07$. The deceleration parameter is now given by

$$
\begin{aligned}
q= & -1-\frac{\dot{H}}{H^{2}}=\frac{1}{2}-\frac{3}{2} \Omega_{D}+\frac{\Omega_{D}^{3 / 2}}{n a} \\
& -\frac{3}{2} b^{2}+\frac{1}{2} \Omega_{k}\left(1-3 b^{2}\right) .
\end{aligned}
$$

Comparing Eqs. (27)-(30) with Eqs. (12), (13), (14) and (17) in the previous section, we see that the scale factor $a$ enters Eqs. (27)-30 explicitly. In the late time where $a \rightarrow \infty$ and $\Omega_{D} \rightarrow 1$, from Eq. (29) with $b^{2}=0$ we have $w_{D} \rightarrow-1$; thus the new agegraphic dark energy mimics a cosmological constant in the late time. Let us now consider the matter-dominated epoch where $a \ll 1$ and $\Omega_{D} \ll 1$. In this case Eq. (28) with $b^{2}=0$ and $\Omega_{k} \ll 1$ approximately becomes

$$
\frac{d \Omega_{D}}{d a} \simeq \frac{\Omega_{D}}{a}\left(3-\frac{2}{n a} \sqrt{\Omega_{D}}\right) .
$$

Solving this equation we find $\Omega_{D}=n^{2} a^{2} / 4$. Substituting this relation into Eq. (29) with $b^{2}=0$, we obtain $w_{D}=-2 / 3$. On the other hand, in the matterdominated epoch, $H^{2} \propto \rho_{m} \propto a^{-3}$. So $\sqrt{a} d a \propto d t=a d \eta$. Thus $\eta \propto \sqrt{a}$. From Eq. (3) we have $\rho_{D} \propto a^{-1}$. Putting this in conservation law, $\dot{\rho}_{D}+3 H \rho_{D}\left(1+w_{D}\right)=0$, we obtain $w_{D}=-2 / 3$. Substituting this $w_{D}$ in Eq. (29) with $b^{2}=0$ and $\Omega_{k} \ll 1$ we find that $\Omega_{D}=n^{2} a^{2} / 4$ as expected. Therefore, all things are consistent. The 
confusion in the original agegraphic dark energy model does not exist in this new model. These results are regardless of the value of $n$. Again one can see that in the absence of interaction between dark energy and dark matter, $b^{2}=0, w_{D}$ in Eq. (29) is always larger than -1 and cannot cross the phantom divide. However, in the presence of interaction, $b^{2} \neq 0$, it is quite possible that $w_{D}$ cross the phantom divide. In the limiting case $\Omega_{k}=0$, Eqs. (28)-(30), restore their respective equations in interacting new agegraphic dark energy model in flat universe [19].

\section{CONCLUSIONS}

There is a wide consensus among cosmologists that the universe has entered a phase of accelerated expansion likely driven by dark energy. However, the nature and the origin of such dark energy is still the source of much debate. Indeed, until now we don't know what might be the best candidate for dark energy to explain the accelerated expansion. Therefore, cosmologists have attended to various models of dark energy by considering all the possibilities they have. In this regard, based on the uncertainty relation of quantum mechanics together with the gravitational effect in general relativity, Cai proposed an agegraphic dark energy model to explain the acceleration of the cosmic expansion [15]. However, the original agegraphic dark energy model had some difficulties. In particular it fails to describe the matter-dominated epoch properly [15]. Thus, Wei and Cai [19] proposed a new model of agegraphic dark energy, while the time scale is chosen to be the conformal time $\eta$ instead of the age of the universe. In this Letter, we extended these agegraphic dark energy models, in the presence of interaction between dark energy and dark matter, to the uni- verse with spatial curvature. Although it is believed that our universe is spatially flat, a contribution to the Friedmann equation from spatial curvature is still possible if the number of e-foldings is not very large [9]. Besides, some experimental data has implied that our universe is not a perfectly flat universe and recent papers have favored the universe with spatial curvature [30]. We obtained the equation of state for interacting agegraphic energy density in a non-flat universe. When the interaction between dark matter and agegraphic dark energy is taken into account, the equation of state parameter of dark energy, $w_{D}$, can cross the phantom divide in the universe with any spacial curvature.

In interacting agegraphic models of dark energy, the properties of agegraphic dark energy is determined by the parameters $n$ and $b$ together. These parameters would be obtained by confronting with cosmic observational data. In this work we just restricted ourselves to limited observational data. Giving the wide range of cosmological data available, in the future we expect to further constrain our model parameter space and test the viability of our model.

Finally I would like to mention that, as we found, the spatial curvature does not seriously modify the qualitative picture of the interacting agegraphic dark energy models but it may however affect the time of onset of the acceleration. For an open universe $\left(\Omega_{k}<0\right)$ the acceleration sets in earlier whereas in a closed universe $\left(\Omega_{k}>0\right)$ the accelerated phase is delayed.

\section{Acknowledgments}

This work has been supported by Research Institute for Astronomy and Astrophysics of Maragha, Iran.
[1] A.G. Riess, et al., Astron. J. 116 (1998) 1009;

S. Perlmutter, et al., Astrophys. J. 517 (1999) 565;

S. Perlmutter, et al., Astrophys. J. 598 (2003) 102;

P. de Bernardis, et al., Nature 404 (2000) 955.

[2] T. Padmanabhan, Phys. Rep. 380 (2003) 235;

P. J. E. Peebles, B. Ratra, Rev. Mod. Phys. 75 (2003) 559 ;

E. J. Copeland, M. Sami and S. Tsujikawa, hepth/060305.

[3] P. Horava, D. Minic, Phys. Rev. Lett. 85 (2000) 1610;

P. Horava, D. Minic, Phys. Rev. Lett. 509 (2001) 138;

K. Enqvist, M. S. Sloth, Phys. Rev. Lett. 93 (2004) 221302

[4] S. D. H. Hsu, Phys. Lett. B 594 (2004) 13.

[5] G. t Hooft, gr-qc/9310026.

L. Susskind, J. Math. Phys. 36 (1995) 6377.

[6] A. Cohen, D. Kaplan, A. Nelson, Phys. Rev. Lett. 82 (1999) 4971.

[7] W. Fischler, L. Susskind hep-th/9806039

[8] M. Li, Phys. Lett. B 603 (2004) 1.
[9] Q. G. Huang, M. Li, JCAP 0408 (2004) 013.

[10] E. Elizalde, S. Nojiri, S.D. Odintsov, P. Wang, Phys. Rev. D 71 (2005) 103504;

B. Guberina, R. Horvat, H. Stefancic, JCAP 0505 (2005) 001;

B. Guberina, R. Horvat, H. Nikolic, Phys. Lett. B 636 (2006) 80;

H. Li, Z. K. Guo, Y. Z. Zhang, Int. J. Mod. Phys. D 15 (2006) 869;

Q. G. Huang, Y. Gong, JCAP 0408 (2004) 006;

J. P. B. Almeida, J. G. Pereira, Phys. Lett. B 636 (2006) 75 ;

Y. Gong, Phys. Rev. D 70 (2004) 064029;

B. Wang, E. Abdalla, R. K. Su, Phys. Lett. B 611 (2005) 21 ;

J. Y. Shen, B. Wang, E. Abdalla, R. K. Su, Phys. Lett. B 609 (2005) 200.

[11] X. Zhang, F. Q. Wu, Phys. Rev. D 72 (2005) 043524;

X. Zhang, Phys. Rev. D 74 (2006) 103505;

X. Zhang, F. Q. Wu, Phys. Rev. D 76 (2007) 023502. 
[12] M. R. Setare, S. Shafei, JCAP 09 (2006) 011;

M. R. Setare, Phys. Lett. B 644 (2007) 99;

M. R. Setare, E. C. Vagenas, Phys. Lett. B 666 (2008)

111

M. R. Setare, Phys. Lett. B 642 (2006) 421;

H. M. Sadjadi, arXiv:0902.2462,

[13] M. R. Setare, Phys. Lett. B 642 (2006) 1.

[14] D. Pavon, W. Zimdahl, Phys. Lett. B 628 (2005) 206;

N. Banerjee, D. Pavon, Phys. Lett. B 647 (2007) 477.

[15] R. G. Cai, Phys. Lett. B 657 (2007) 228.

[16] F. Karolyhazy, Nuovo.Cim. A 42, 390 (1966);

F. Karolyhazy, A. Frenkel and B. Lukacs, in Physics as natural Philosophy edited by A. Shimony and H. Feschbach, MIT Press, Cambridge, MA, (1982);

F. Karolyhazy, A. Frenkel and B. Lukacs, in Quantum Concepts in Space and Time edited by R. Penrose and C.J. Isham, Clarendon Press, Oxford, (1986).

[17] M. Maziashvili Int. J. Mod. Phys. D 16 (2007) 1531;

M. Maziashvili, Phys. Lett. B 652 (2007) 165.

[18] H. Wei and R. G. Cai, Eur. Phys. J. C 59 (2009) 99.

[19] H. Wei and R. G. Cai, Phys. Lett. B 660 (2008) 113.

[20] J. Cui, et al., arXiv:0902.0716.

Y. W. Kim, et al., Mod. Phys. Lett. A 23 (2008) 3049;

Y. Zhang, et al. arXiv:0708.1214,

J .P Wu, D. Z. Ma, Y. Ling, Phys. Lett. B 663, (2008) 152 ;

K. Y. Kim, H. W. Lee, Y. S. Myung, Phys.Lett. B 660 (2008) 118;

J. Zhang, X. Zhang, H. Liu, Eur. Phys. J. C 54 (2008) 303;

I. P. Neupane, Phys. Lett. B 673 (2009) 111.

[21] A. Sheykhi, arXiv:0907.5144,

A. Sheykhi, 0908.0606.

[22] M. R. Setare, arXiv:0907.4910

M. R. Setare, arXiv:0908.0196.

[23] H. Wei and R. G. Cai, Phys. Lett. B 663 (2008) 1;

X. Wu, et al., arXiv:0708.0349.

[24] L. Amendola, Phys. Rev. D 60 (1999) 043501;

L. Amendola, Phys. Rev. D 62 (2000) 043511;

L. Amendola and C. Quercellini, Phys. Rev. D 68 (2003) 023514 ;

L. Amendola and D. Tocchini-Valentini, Phys. Rev. D 64 (2001) 043509 ;

L. Amendola and D. T. Valentini, Phys. Rev. D 66 (2002)
043528.

[25] W. Zimdahl and D. Pavon, Phys. Lett. B 521 (2001) 133;

W. Zimdahl and D. Pavon, Gen. Rel. Grav. 35 (2003) 413;

L. P. Chimento, A. S. Jakubi, D. Pavon and W. Zimdahl, Phys. Rev. D 67 (2003) 083513.

[26] M. R. Setare, Eur. Phys. J. C 50 (2007) 991;

M. R. Setare, JCAP 0701 (2007) 023;

M. R. Setare, Phys. Lett. B 654 (2007) 1;

M. R. Setare, Phys. Lett. B 642 (2006) 421.

[27] B. Wang, Y. Gong and E. Abdalla, Phys. Lett. B 624 (2005) 141;

B. Wang, C. Y. Lin. D. Pavon and E. Abdalla, Phys. Lett. B 662 (2008) 1.

[28] G. Olivares, F. Atrio, D. Pavon, Phys. Rev. D 71 (2005) 063523.

[29] B. Wang, C. Y. Lin and E. Abdalla, Phys. Lett. B 637 (2005) 357;

M. R. Setare, Eur. Phys. J. C 52 (2007) 689;

Bin Wang, et al., astro-ph/0607126

[30] C. L. Bennett, et al., Astrophys. J. Suppl. 148 (2003) 1; D. N. Spergel, Astrophys. J. Suppl. 148 (2003) 175;

M. Tegmark, et al., Phys. Rev. D 69 (2004) 103501;

U. Seljak, A. Slosar, P. McDonald, JCAP 0610 (2006) 014 ;

D. N. Spergel, et al., Astrophys. J. Suppl. 170 (2007) 377.

[31] J. L. Sievers, et al., Astrophys. J. 591 (2003) 599; C.B. Netterfield, et al., Astrophys. J. 571 (2002) 604;

A. Benoit, et al., Astron. Astrophys. 399 (2003) L25;

A. Benoit, et al., Astron. Astrophys. 399 (2003) L19.

[32] J. P. Uzan, U. Kirchner, G.F.R. Ellis, Mon. Not. R. Astron. Soc. 344 (2003) L65;

A. Linde, JCAP 0305 (2003) 002;

M. Tegmark, A. de Oliveira-Costa, A. Hamilton, Phys. Rev. D 68 (2003) 123523;

G. Efstathiou, Mon. Not. R. Astron. Soc. 343 (2003) L95; J. P. Luminet, J. Weeks, A. Riazuelo, R. Lehou, J. Uzan, Nature 425 (2003) 593;

G. F. R. Ellis, R. Maartens, Class. Quantum Grav. 21 (2004) 223.

[33] R. R. Caldwell, M. Kamionkowski, astro-ph/0403003,

B. Wang, Y. G. Gong, R. K. Su, Phys. Lett. B 605 (2005) 9.

[34] S. Tsujikawa, M. Sami, Phys. Lett. B 603 (2004) 113.

[35] M. Doran, J. Jackel, Phys. Rev. D 66 (2002) 043519. 